\title{
The effects of reinforcement frequency on performance in a multiple fixed-ratio schedule*
}

\author{
MARK R. ZASLAV and JOHN J. PORTERं \\ Lniversin of I'isconsin. Wilwaukee. I'isconsin 53201
}

\begin{abstract}
A barpress analog to the double-alley runway was sought by varying percentage reward in the first of two consecutive FR 18s. Groups of six rats each were given $0 \dot{r}_{c}, 5()_{c}$, or $100 r_{c}$ reinforcement upon completion of the first FR 18 ; after a $5-\mathrm{sec}$ midtrial interval. the second FR 18 was administered on a separate lever and all groups received CRF reward upon its completion. Group 50 Ss performed faster after nonreward than after reward. Group 50 Ss performed faster after nonreward than did $0{ }_{r}$ Ss. A measure of midtrial behavior revealed a difference between groups in orienting to the bars. When all groups were shifted to a 50 , first component schedule (Phase II), there were no statistically reliable effects of prior reinforcement history on rewarded or nonrewasded responding. The Phase 1 results were taken to demonstrate a frustration effect similar to that of the double alley.
\end{abstract}

As Amsel. Mackinnon. Rashotte, and Surridge (1964) have noted. a discrete-trials leverpressing demonstration of the double-alley frustration effect (cf. Amsel. 1958: Wagner, 1959) would be desirable from both theoretical and methodological standpoints.

Hughes and Dachowski (1973), in discussing three attempts at operant analogs to the double-alley runway (Hamm \& Zimmerman. 1967: Platt \& Senkowski. 1970: Cole \& Van Fleet. 1970), concluded that none of these studies could be interpreted as supportive of a frustration analysis. In their own study, several procedural manipulations resulted in the detection of apparent rate-indexed frustration effects in an operant setting. Thus. Hughes \& Dachowski (1973) used rats in a discrete-trial double-lever procedure in which completion of an FR 4 response chain on the first bar produced either reinforcement or nonreinforcement; $15 \mathrm{sec}$ later the second bar entered the chamber, and completion of an FR 4 here produced reinforcement for all animals. An experimental group received $50 \%$ random reward on the first lever, while a control group never received reinforcement on the first lever. Second bar response rate was significantly faster after nonreward on the first bar than after reward on the first bar. Second bar performance after nonreward by the experimental group exceeded that of control group second bar rates, thus arguing against a response depression interpretation of the difference within the experimental group (Platt \& Senkowski. 1970).

In the present experiment several manipulations were introduced in order to increase the magnitude of frustration effects and to allow more detailed analysis in

*This paper is based upon a thesis submitted by the first author to the University of Wisconsin-Milwaukee in partial fulfillment of the requirements for a Master of Science degree under the direction of the second author.

tRequests for reprints should be sent to John J. Porter, Department of Psychology, University of Wisconsin, Milwaukee, Wisconsin 53201. terms of standard instrumental conditional paradigms. Toward this end, two consecutive FR 18 chains were used on separate response levers. The two chains were separated by a short midtrial interval (MTI) of $5 \mathrm{sec}$ and each pair by an intertrial interval (ITI) of $2 \mathrm{~min}$. The short MTI was chosen in order to make the second chain more sensitive to first chain reinforcement events, while the long ITI was used in order to make successive trials more discriminable. Following a suggestion by Amsel et al (1964) and a procedure of Hughes and Dachowski (1973), each 18-response component was split for analysis into successive units of 6 responses, which roughly corresponded to start, run, and goal speeds, respectively. It was hoped that any characteristic gradients within a chain component could be compared to those occurring in the double alley. At the end of the first component, Ss received $0 \%, 50 \%$, or $100 \%$ reward. The three groups were included in order to yield a complete analog to the double-alley work by Wagner (1959).

\section{METHOD \\ Subjects}

Eighteen Holtzman albino male rats, approximately 130 days old when training began, were randomly assigned to three groups of equal size.

\section{Apparatus}

One $17.5 \times 20 \mathrm{~cm}$ test chamber was used. Two Lehigh Valley retractable levers were located $10 \mathrm{~cm}$ apart on one wall of the chamber, with a metal foodcup located midway between them. A small pilot stimulus lamp was located $3 \mathrm{~cm}$ above each lever. A Davis PD 109 feeder delivered 97-mg Noyes pellets to the foodcup. A Grason-Stadler E4690A drinkometer was connected to the metal foodcup and recorded the number of cup touches during the MTI. The test chamber was illuminated by a $7.5-\mathrm{W}$ houselight and was enclosed in a sound-resistant ventilated chest that was housed in an IAC 402-A audiometric chamber. Electromechanical equipment was used for programming the experimental sequences and data were recorded by BRS POC-112 printout counters. 


\section{Design}

The study consisted of two phases. During the 8 days of Phase I, Ss were assigned to groups that received $0 \%, 50 \%$, or $100 \%$ reinforcement at the end of the first chain and $100 \%$ reinforcement at the end of the second chain. During the following 3 days, Phase II, all Ss received $50 \%$ reward at the end of the first chain and continued to receive $100 \%$ reward at the completion of Chain 2. Each chain component was associated with distinctive cue lights. Twenty trials were conducted daily. Following the procedures of Davenport, Flaherty, and Dryud (1966), Davenport and Thompson (1965), and Carlson (1968), the MTI was $5 \mathrm{sec}$, during which both levers were retracted, and the ITI was $2 \mathrm{~min}$. The use of a longer ITI than MTI and separate bars was intended to parallel the respective between- and within-trial discreteness encountered in the typical double-alley runway situation. Reinforcement was one $97-\mathrm{mg}$ Noyes pellet throughout.

\section{Procedure}

Upon arrival, Ss were placed upon ad lib feeding for 5 days. On Day 6 Ss were placed on a 22-h food-deprivation schedule that continued throughout the experiment. Starting $15 \mathrm{~min}$ after each experimental session, animals were given $45 \mathrm{~min}$ free access to food. On Day 12 pretraining began with 2 days of adaptation to the test chamber, $1 \mathrm{~h} / \mathrm{day}$, during which response levers were retracted, the houselight on, and the stimulus lamps off. On Day 14 Ss were magazine trained, each receiving 60 reinforcements on a VI $30-\mathrm{sec}$ schedule. On Day 15 the right response lever was inserted into the chamber and all $S$ s were shaped to a CRF requirement, where each barpress yielded one 97-mg Noyes pellet. On Day 16 an FR 6 requirement was introduced on the right lever, with each animal receiving approximately 65 reinforcements. On Day $17 \mathrm{Ss}$ were placed on the following schedule: The left response lever entered the chamber simultaneously with the onset of the left stimulus lamp and houselight. When $S$ completed an FR 6 , one pellet was delivered, the left stimulus lamp was extinguished, and the left lever was retracted. Five seconds later, the right lever was introduced and the right stimulus lamp lit. Completion of FR 6 produced another pellet, turned off the right stimulus lamp, and retracted the right lever. This sequence was repeated as a trial, 20 trials being conducted using an ITI of $2 \mathrm{~min}$.

On Day 18 the same sequence was repeated with two variations. First, all Ss had to complete an FR 12 on each lever to terminate the component. Second, the completion of an FR 12 on the left lever led to Group 100 receiving one Noyes pellet, Group 0 not receiving a pellet, and Group 50 receiving a pellet on a random $50 \%$ of the trials, the other $50 \%$ being nonrewarded. Immediately after the completion of the right lever component, all Ss were reinforced $100 \%$ of the time. This sequence constituted a trial, 20 trials being presented at an ITI of $2 \mathrm{~min}$.

On Day 19 Phase I of experimental training began. Sequence of bar presentation, midtrial interval (time between left lever retraction and right lever insertion), and intertrial interval remained the same as for the previous day of pretraining. The response requirement on each lever was increased to 18 . Once again, completion of the response requirement on the left lever produced either reward or nonreward, depending upon group, with Groups 100,0 , and 50 receiving a pellet on all, none, or a random $50 \%$ of the trials, respectively. Again, all groups received a pellet after completing the response requirement on the right lever. Twenty-one trials per day were run under these conditions, with the first trial of the day constituting a warm-up period when data was not recorded. Times to complete three successive groups of six responses on both levers were recorded. In addition, latency to the first barpress after right lever insertion was recorded as well as the number of touches to the foodcup during the MTI. Phase I continued for 8 day's (160 trials).
Phase II began on Day 27. All aspects of the procedure were the same as durng Phase I, except that Groups 100 and 0 were shifted to the $50 \%$ schedule of reinforcement on the left lever previously adminisiered to Group 50 . Group 50 was maintained on its 50\% schedule during Phase II, which lasted 3 days $(60$ trials) and terminated the experiment.

\section{RESULTS AND DISCUSSION}

Mean daily speeds (1/latency) were computed for each session of 20 trials. The mean speeds for each six responses on the left lever (first component) and on the right lever (second component) will be referred to as start (first 6 responses), run (Responses 7-12), and goal (Responses 13-18) speeds. Because frustrative effects were expected in the second FR 18 component, these data will be considered first.

\section{Phase I: Second Component}

In analyzing the results for the second component, separate means were derived from Group 50 depending upon whether the trial included a reinforced first component ( $\mathrm{R}$ ) or a nonreinforced first component $(\mathrm{N})$ (cf. McHose, 1963: Wagner, 1959). Within-Ss analyses were performed on Group 50 after $\mathrm{R}$ or $\mathrm{N}$ first component. Separate analyses of variance were performed comparing Groups 100, 0, and Group 50 performance on $\mathrm{R}$ trials in one case and Groups 100,0 , and Group 50 performance on $\mathrm{N}$ trials in the other (cf. McHose, 1963). These two kinds of between-groups analyses will be reported for each variable in the aforementioned order.

A measure of number of foodcup touches was included to reveal any systematic differences among groups in MTI behavior. Percentage reinforcement (PR) did result in different activity patterns during MTI. Within-Ss analysis on Group 50 demonstrated that Ss made significantly fewer cup touches after nonreward $[F(1.80)=34.30, p<.01]$. Turning to the between-groups analyses, there was a significant difference in foodcup touches during MTI as a function of PR between Groups 100, 0, and $50(R)[F(2,15)=$ $10.44, \mathrm{p}<.01]$ and Groups 100,0 , and $50(\mathrm{~N})[\mathrm{F}(2,15)$ $=4.78, p<.01]$. Both analyses revealed a change in number of cup contacts over days (D) $[F(7,105)=2.01$, $p<.05$ and $F(7,105)=5.47, p<.01$, respectively]. The analysis between Groups 100,0 , and 50 on $\mathrm{N}$ trials yielded a significant PR by $D$ interaction $[F(14,105)=$ $2.76, \quad p<.01]$, apparently due to Group 50(N) exhibiting a more marked decrease in foodcup touches over sessions than any of the other groups. Group 0 maintained a relatively stable low number of contacts. while Group 100 and Group 50 Ss on R trials made significantly more contacts over all 8 days. The foodcup data indicated that Group 0 Ss completed the first component and immediately attended to the insertion of the right lever, without much intervening foodcup activity. Group 100 and Group 50 Ss on R trials. of 
course, attended to the foodcup during the MTI while consuming their first component reward. While Group 50 animals on $\mathrm{N}$ trials gradually learned over trials not to approach the foodcup in the absence of magazine stimuli and to attend instead to right lever insertion.

In agreement with an assertion by Hughes and Dachowski (1973), it appeared that this pattern of MTI responding directly influenced second component response latency. The within-Ss analysis over Group 50 confirmed significantly shorter latencies on $\mathrm{N}$ trials. as seen in Fig. $1[F(1.80)=65.94 . p<.01]$. Figure 1 further reveals that Group $0 \mathrm{Ss}$ and Group 50 Ss on $\mathrm{N}$ trials both exhibited similar short latencies to first right lever barpress, while Group 100 and Group 50 Ss on R trials showed consistently longer latencies over days. These observations were confirmed by $P R$ effects $[F(2,15)=10.44, p<.01$ and $F(2.15)=4.78 . p<.05$, respectively]. Tukey`s HSD tests (Kirk. 1968) revealed that Group 100 and Group 50 Ss on R trials exhibited significantly longer latencies than either Group 0 or Group 50 Ss on $N$ trials $(p<.05)$. The notion of foodcup activity as a competing response to orientation toward the right lever would seem adequate to explain the agreement between the foodcup touch and right lever latency data. It may be noted that the latency data from the present study agree with Hughes and Dachowski's (1973) start results. representing virtually the same measure of responding.

Results for the start speed measure are shown in the left panel of Fig. 2. The within-Ss analysis for Group 50 revealed reward condition as the only significant factor $[F(1,80)=14.15, p<.01]$. Both between-groups analyses revealed significant $P R$ effects $[F(2,15)=$ $11.62, p<.01$ and $F(2,15)=7.26, p<.01$, respectively]. The analysis containing Group $50 \mathrm{Ss}$ on $\mathrm{N}$ trials yielded an additional significant effect of $D$ $[F(7,105)=5.95, p<.01]$ and a significant $P R$ by $D$ interaction $[F(14,105)=2.70, p<.01]$. It would appear from Fig. 2 that Group 50 on $\mathrm{N}$ trials was responsible for the $D$ and $P R$ by $D$ effects. Thus, after Day 5, Group 50 Ss on $N$ trials were faster than Group 0 , but this superiority was not significant ( $>.05)$. Although not statistically reliable, this crossover suggested a frustrative increment for Group 50 Ss on $\mathrm{N}$ trials in start performance.

For run speed, shown in the center panel of Fig. 2, Groups 100 and 0 were much closer in performance. This finding is in accord with the fact that MTI consummatory activity did not exert a direct influence on run speed. Both between-groups analyses yielded a significant increase over days $[F(7,105)=11.04, p<.01$ and $F(7,105)=16.87, p<.01$, respectively]. Only the between-groups $50(\mathrm{~N}), 100$, and 0 analysis revealed a significant $P R$ by $D$ interaction $[F(14,105)=1.86$, $\mathrm{p}<.05]$. Simple effects of $P R$ were significant on Day 8 $[F(2,120)=3.13, p<.05]$. A Tukey HSD test was performed comparing group means from Day 8 .

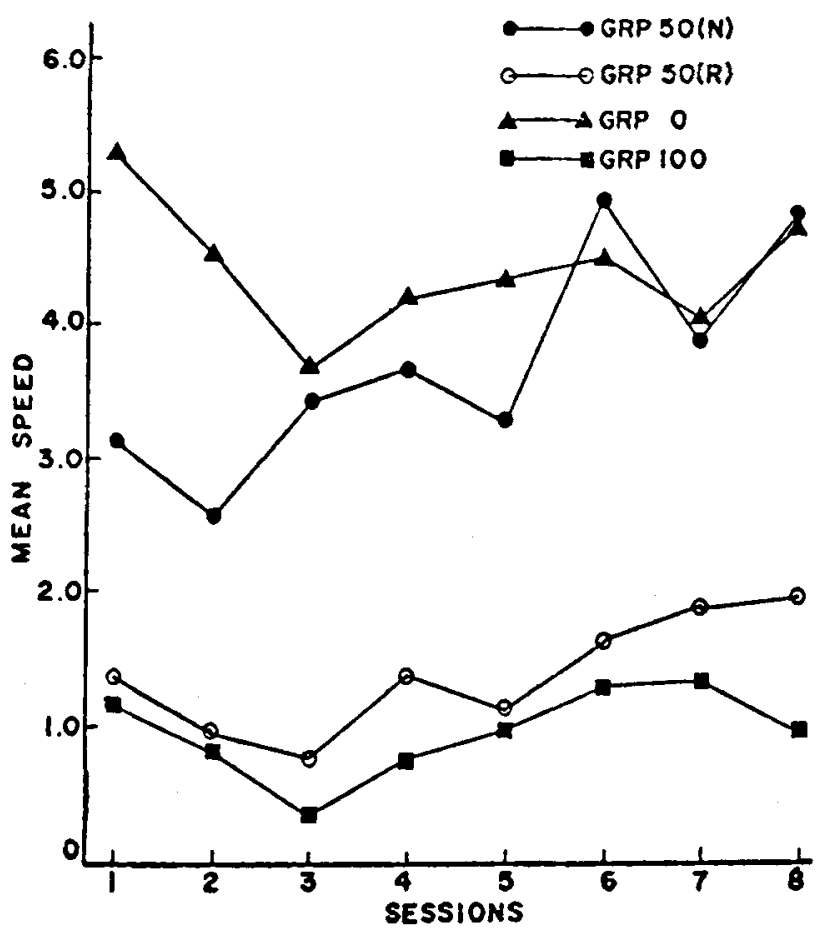

Fig. 1. Mean reciprocal times to first lever response over the

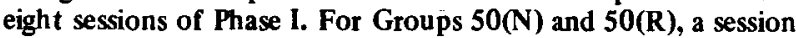
represents 10 trials, while for Groups 100 and 0 , a session includes 20 trials. This applies to Fig. 2 as well.

Group 50 Ss on $\mathrm{N}$ trials performed significantly faster than Group $0 \quad(p<.05)$, with no other group comparisons attaining significance. The within-Ss analysis on Group 50 revealed significantly faster speeds after nonreward $[F(1,80)=10.71, p<.01]$. In sharp contrast to the behavior of $0 \%$ and $100 \%$ controls, Group $50 \mathrm{Ss}$ on $\mathrm{N}$ trials exhibited a very definite increase over days until, from Day 5 on, these animals' performance exceeded that of Group 0 , significantly so by Day 8 . It was evident that reward expectancy for Group 50 grew until, by Day 5 , it was sufficient for nonreward to result in measurable frustrative facilitation relative to Group 0. Data from the run speed measure, thus, offer evidence of frustrative energization manifested by Group 50 Ss after nonreward, relative to both their own performance after reward and to Group 0. Similarly, Hughes and Dachowski (1973) obtained a significant frustration effect relative to chronic nonrewarded controls only in their midchain measure (run speed being the present study's equivalent measure).

Data for the goal speed measure are presented in Fig. 2c. Although both between-groups analyses revealed a significant increase over days $[\mathrm{F}(7,105)=12.91$, $\mathrm{p}<.01$ and $\mathrm{F}(7,105)=15.96, \mathrm{p}<.01$, respectively $]$ there were no significant effects of PR. The within-Group 50 analysis also yielded only days as significant $[F(1,80)=9.17, p<.01]$. The lack of other effects here may well be due to the fact that all groups 


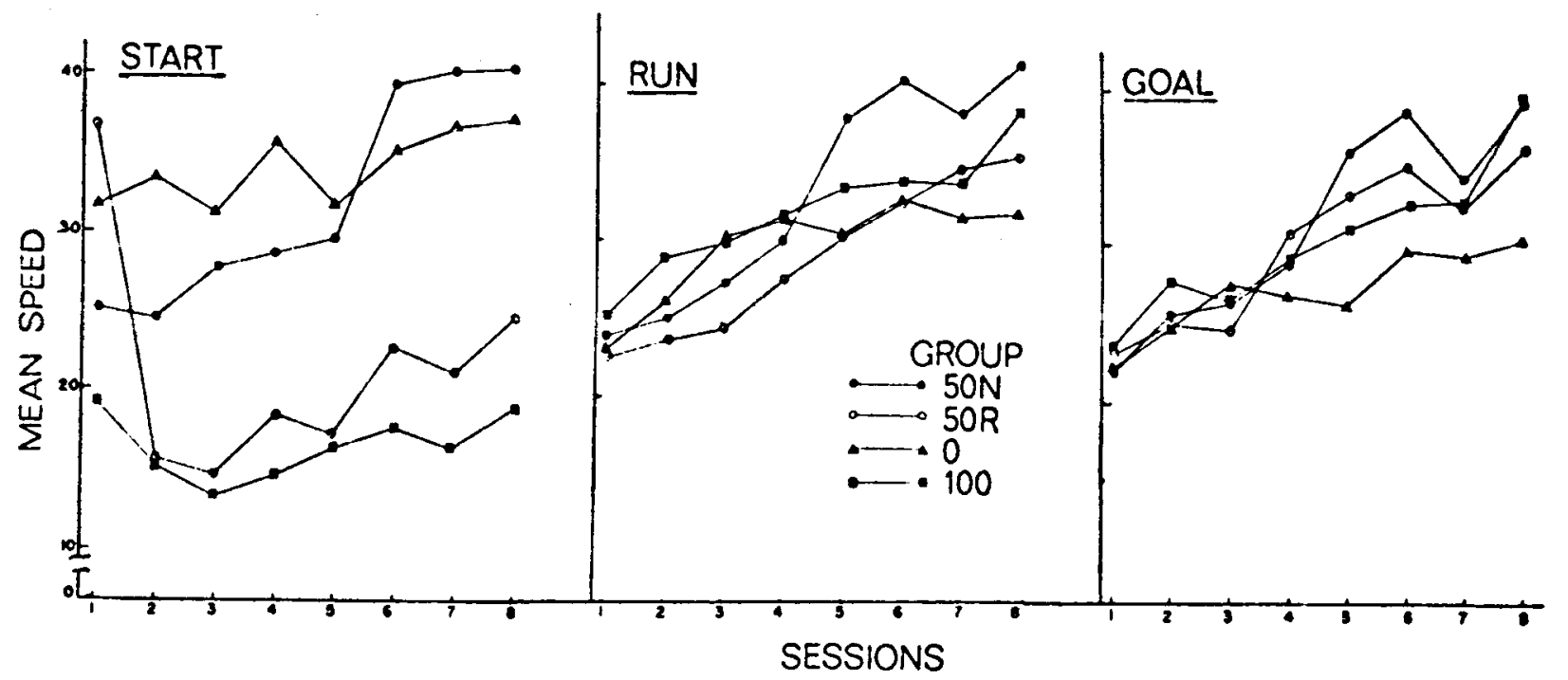

Fig. 2. Mean reciprocal times to complete successive sets of six responses on the right lever over the eight sessions of Phase $I$. Left panel: start speed; center panel: run speed; right panel: goal speed.

were responding in this measure to the same terminal reward, with the effects of second component reward able to obscure any more subtle group effects. Hughes and Dachowski (1973) also failed to obtain group effects in the goal section of their chain.

\section{Phase I: First Component}

Separate analyses of variance were performed for start, run, and goal measures of the first component in Phase I. All analyses revealed a significant effect of groups $[F(2,15)=9.71, p<.01 ; F(2,15)=6.01$, $p<.01$; and $F(2,15)=10.92, p<.01$ for start, run, and goal speed measures, respectively]. In addition, there was a significant increase in speed over days $[F(7,105)=$ $3.09, \mathrm{p}<.05]$ in the goal speed measure. Tukey's HSD test revealed that Group 100 responded significantly faster than Groups 50 and 0 in all three measures $(p<.05)$. It was apparent from these data that incentive at the end of the first component operated on rate of responding in the expected direction. Thus, Group 100 animals, responding for a food pellet at the end of the first component, performed faster than Group 0 and Group 50 Ss that responded for no pellet or one pellet on $50 \%$ of the trials. This pattern held across all three measures of first component responding.

\section{Phase II}

In Phase II animals in all groups were shifted to a 50\% first component reward schedule, and each group was differentiated as to whether it received an $\mathrm{R}$ or an $\mathrm{N}$ trial. Because they are most critical with respect to a frustration analysis, only the second component measures of responding will be presented.

Start speed analysis yielded only reward outcome as significant $[F(1,15)=90.43, p<.01]$. The left panel of
Fig. 3 shows that Groups 0 and 100 on nonrewarded trials exhibited similar rates in the start measure, both being somewhat slower than Group $50 \mathrm{Ss}$ after nonreward.

Results for the run speed measure are shown in the center panel of Fig. 3. Here run speed, being free of the influence of MTI behavior, revealed Group 100 Ss faster than Group 50 animals after nonreward. Group 50 and 100 nonrewarded performance exceeded that of Group 0 after nonreward. Analysis of variance revealed only reward condition as significant $[F(1,15)=37.71$, $\mathrm{p}<.01]$, although the effect of reinforcement history approached but did not attain significance $[F(2,15)=$ $3.08, p>.05]$.

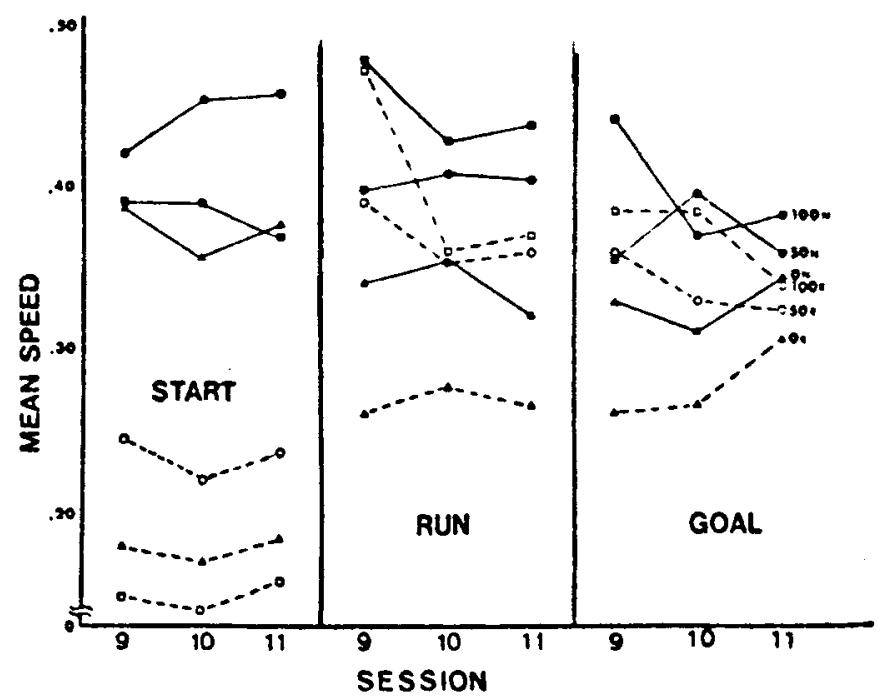

Fig. 3. Mean reciprocal times to complete successive sets of six responses on the right lever over the three sessions of Phase II. Left panel: start speed : center panel: run speed: right panel: goal speed. 
For goal speed, shown in the right panel of Fig. 3. reward condition again proved to be the only significant factor $[F(1,15)=10.83, p<.01]$. On the basis of results in Phase I and from the very similar findings of Hughes and Dachowski (1973) where frustrative effects were detected only in the midchain measure of responding, we might look for the effects of prior reinforcement history, if they occurred at all. to be manifested in the run measure of Phase II responding. Frustration theory would have predicted that Group 100 Ss, having reached asymptotic reward expectancy (160 rewarded trials in Phase I), should have evidenced strong and immediate frustrative facilitation upon exposure to nonreward. Group 0 animals, on the other hand, should have shown a smaller facilitation, because reward expectancy was presumably low after Phase I as a consequence of the chronically nonrewarded first component. Instead, all three Phase II measures failed to reveal any statistically reliable effects of prior reinforcement history in the first component or interactions between reinforcement history and reward condition.

\section{SUMMARY AND CONCLUSIONS}

The present study and that of Hughes and Dachowski employed procedures designed to parallel the double alley and, unlike previous alley analog studies, obtained more traditional alley effects. Several procedural differences exist between the current study and those of Platt and Senkowski (1970) and Hughes and Dachowski (1973). Hughes and Dachowski (1973), Platt and Senkowski (1970), and the present study employed MTIs of 15,10 , and $5 \mathrm{sec}$, respectively. ITIs in these respective studies were 15,40 , and $120 \mathrm{sec}$. Similarly, response requirements were FR 4, FR 8, and FR 18. It will be noted that Platt and Senkowski (1970) failed to obtain frustration effects while using values of the procedural variates mentioned that were intermediate to those used by both the present investigators and Hughes and Dachowski (1973). Since the latter studies achieved frustration effects, the failure of the Platt and Senkowski (1970) study to obtain such effects is more reasonably attributed to their reliance on a single leyer than any other procedural variation. In addition, the recording of foodcup touches during $\mathrm{MTl}$ and the segmenting of the response chains permitted a more detailed analysis of the experimental effects achieved.

In the first component in Phase 1 , Platt and Senkowski failed to obtain any difference between Groups 0,50 , and 100, whereas in the present study and in Hughes and Dachowski's there were clear group differences. Again, it would appear that Piatt and Senkowski's single lever was the chief reason for their animals failure to make chain discriminations.

The general conclusion emerging from the present study is that a barpress analog to the double-alley frustration paradigm seemed to produce frustration effects, with the additional indication that traditional control groups, such as the Wagner control, are more subject to competing consummatory influences than seems to be the case in the alley. The barpress situation seems especially sensitive to instances where consummatory and barpress responses are incompatible. That operant frustration effects can be of considerable magnitude is attested to by their appearance in the present study despite the noted elevation of the Wagner control group and the relatively small number of Ss in each group $(\mathrm{N}=6)$. The robust nature of operant frustration effects is demonstrated in their detection in both the present study and that by Hughes and Dachowski, despite several procedural differences.

\section{REFERENCES}

Amsel, A. The role of frustrative nonreward in noncontinuous reward situations. Psychological Bulletin, 1958, 55, 102-119. Amsel, A, MacKinnon, J. R., Rashotte, M. E., \& Surridge, C. T. Partial reinforcement (acquisition) effects within subjects. Journal of the Experimental Analysis of Behavior, 1964, 7, 135-138.

Carlson, J. G. Frustrative nonreinforcement of operant responding: Magnitude of reinforcement and response force effects. Psychonomic Science, 1968, 11, 307-308.

Cole, M., \& Van Fleet, F. The frustration effect as a function of interoceptive and exteroceptive cues in a Skinner box analogue of the double runway. Psychonomic Science, 1970, $20,33-35$.

Davenport, J. W., Flaherty, C. F., \& Dryud, J. P. Temporal persistence of frustration effects in monkeys and rats. Psychonomic Science, 1966,6, 411412.

Davenport, J. W., \& Thompson, C. I. The Amsel frustration effect in monkeys. Psychonomic Science, 1965, 3, 481-482.

Hamm, H., \& Zimmerman, J. The frustration effect in operant studies: A necessary control procedure. Psychonomic Science, $1967,9,253-254$.

Hughes, L. F., \& Dachowski, L. The role of reinforcement and nonreinforcement in an operant frustration effect. Animal Learning \& Behavior, 1973, 1, 68-72.

Kirk, R. E. Experimental design: Procedures for the behavioral sciences. Belmont, Calif: Wadsworth, 1968.

McHose, J. H. Effect of continued reinforcement on the frustration effect. Journal of Experimental Psychology, 1963, $65,444-450$.

Platt, J. R., \& Senkowski, P. C. Effects of discrete-trials reinforcement frequency and changes in reinforcement frequency on preceding and subsequent fixed-ratio performance. Journal of Experimental Psychology, 1970, 85, 95-104

Wagner, A. R. The role of reinforcement and nonreinforcement in an "apparent frustration effect." Journal of Experimental Psychology, 1959, 57, 130-136.

(Received for publication February 1, 1973; revision received October 1,1973 .) 